\title{
Cognitive functioning in children with Prader-Willi syndrome during 8 years of growth hormone treatment
}

\author{
S H Donze ${ }^{1,2}$, L Damen ${ }^{1,2}$, E F Mahabier ${ }^{1}$ and A C S Hokken-Koelega ${ }^{1,2}$ \\ ${ }^{1}$ Dutch Growth Research Foundation, Rotterdam, The Netherlands and ${ }^{2}$ Erasmus University Medical Centre/Sophia \\ Children's Hospital, Department of Paediatrics, Subdivision of Endocrinology, Rotterdam, The Netherlands
}

Correspondence should be addressed to S H Donze Email

S.Donze@kindengroei.nl

\begin{abstract}
Objective: Children with Prader-Willi syndrome (PWS) have mild to moderate cognitive impairment. Short-term studies showed positive effects of growth hormone (GH) on cognitive development. This study investigated the effects of 8 years of GH on cognitive development in children with PWS. We also investigated whether starting GH during infancy results in higher cognitive functioning after 8 years of $\mathrm{GH}$.

Design: Longitudinal study in 43 children with PWS during 8 years of GH (median age at GH start 8.1 years). Cognitive functioning after 8 years was compared to another group of 22 children with PWS (median age at GH start 1.4 years). Methods: Cognitive functioning was measured by Wechsler Intelligence Scale for Children. Vocabulary, Similarities and Block Design subtests were expressed as standard deviation scores (SDS) and total IQ (TIQ) calculated.

Results: Estimated mean $(95 \% \mathrm{Cl})$ Block Design SDS changed from $-2.2(-2.6 ;-1.8)$ at GH start to $-1.8(-2.2 ;-1.4)$ after 8 years of $\mathrm{GH}(P=0.18)$, similarly SDS from $-1.5(-2.1 ;-0.9)$ to $-1.3(-1.9 ;-0.7, P=0.66)$ and TIQ from $66(60 ; 72)$ to $69(63 ; 75, P=0.57)$. Vocabulary SDS remained similar, being $-1.9(-2.3 ;-1.4)$ at GH start and $-1.9(-2.4 ;-1.5)$ after 8 years $(P=0.85)$. After 8 years of GH Vocabulary, SDS and TIQ were higher in the children who started GH during infancy, compared to those who started GH later in childhood $(P<0.01, P=0.04$, respectively).

Conclusions: Cognitive functioning in children with PWS remains similar during long-term GH and develops at the same pace as healthy peers.
\end{abstract}

\section{Introduction}

Prader-Willi syndrome (PWS) is a rare genetic disorder resulting from the loss of expression of locus q11-q13 (PWS region) on the paternally derived chromosome 15. This loss of expression is mostly due to a paternal deletion or maternal uniparental disomy (mUPD) and leads to a characteristic phenotype, including muscular hypotonia, short stature, hyperphagia with an increased risk of obesity, psychomotor delay, neurobehavioral problems and cognitive impairment (1). Patients with PWS typically have mild to moderate cognitive impairment with an average IQ between 60 and $70(2,3)$.
Growth hormone $(\mathrm{GH})$ is a registered treatment for children with PWS since 2000. The physical benefits have been thoroughly investigated and include an improvement in body composition, linear growth and physical strength $(4,5,6,7)$. GH treatment is also associated with cognitive benefits, which are attributed to the effects of GH and insulinlike growth factor (IGF-I) on brain growth, development and myelinisation $(8,9)$. A recent review about the effects of GH treatment on cognition concluded that GH could stimulate GH receptors in brain areas involved in learning and memory, thereby improving cognitive functioning (10).

Published by Bioscientifica Ltd. 
In PWS, few studies have investigated the effects of $\mathrm{GH}$ on cognitive functioning. Findings were not unequivocal, with some studies reporting an improvement in psychomotor development and cognition $(2,6,11$, 12 , 13), while others describe no cognitive benefits of $\mathrm{GH}(14,15)$. We previously demonstrated in a 2-year randomized controlled trial that cognitive functioning in GH-treated children with PWS developed at the same pace as cognitive functioning in healthy references, while abstract verbal reasoning and vocabulary significantly deteriorated in untreated controls (6). Furthermore, in 50 children with PWS who started GH at a mean age of 7.8 years, we found a significant improvement in abstract verbal reasoning and visuospatial skills during 4 years of $\mathrm{GH}$ treatment (2). Most of these studies evaluated shortterm effects of GH in children with PWS and there are currently no studies investigating the effects of more than 4 years of GH treatment in children with PWS.

In this prospective study, we therefore investigated the longitudinal effects of 8 years of GH treatment on cognitive functioning in children with PWS from our Dutch PWS cohort. We also investigated whether starting GH treatment during infancy, that is, before the age of 2 years, would result in higher cognitive functioning after 8 years of GH compared to starting GH later in childhood. We hypothesized that cognitive functioning would not deteriorate during long-term GH treatment and would progress at the same pace as healthy references. Additionally, we hypothesized that starting GH during infancy could benefit cognitive functioning more than starting GH later in childhood.

\section{Subjects and methods}

\section{Subjects}

A longitudinal, prospective cohort study was performed in 43 children with PWS to investigate the effects of 8 years of GH on cognitive functioning. All children were selected from our Dutch PWS Cohort based on the following criteria: (1.) genetically confirmed diagnosis of PWS, (2.) naive to GH treatment at time of enrolment, (3.) at least 3 Wechsler Intelligence Scale for Children (WISC) tests performed during 8 years of GH. Thirty-five of 43 children participated in our previous study investigating cognitive development in children with PWS during 4 years of GH treatment (2).

In addition, a cross-sectional study was performed to investigate whether starting $\mathrm{GH}$ treatment during infancy, that is, before the age of 2 years, would result in improved cognitive functioning after 8 years of $\mathrm{GH}$. We compared WISC results after 8 years of GH of a separate group of 22 children with PWS from our Dutch PWS Cohort who started GH treatment during infancy to the 43 longitudinally studied children who started GH later in childhood. We could not evaluate the longitudinal effects of 8 years of $\mathrm{GH}$ on cognitive functioning in the first group, because WISC is not suitable for children younger than 6 years of age. After 8 years of continuous GH treatment, all 22 children who started GH during infancy were older than 6 years and their cognitive functioning could be tested by WISC. The study protocol was approved by the Medical Ethics Committee of the Erasmus Medical Centre (Rotterdam, The Netherlands) and the collaborating centres. Written informed consent was obtained from parents and from children older than 12 years; assent was obtained from children younger than 12 years.

\section{Design}

In this prospective cohort study, all participants were followed at the Dutch PWS Reference Centre in collaboration with paediatric endocrinologists and paediatricians in other hospitals in the Netherlands. Children were treated with Genotropin (Pfizer), administered subcutaneously once daily at bedtime with a dose of $1 \mathrm{mg} / \mathrm{m}^{2} /$ day ( $\approx 0.035 \mathrm{mg} / \mathrm{kg} /$ day) for 8 consecutive years. All children were GH naive at the start of the study. The GH dose was regularly adjusted based on calculated body surface area and serum IGF-I levels.

During the study, all children were seen every 3 months for anthropometric measurements by the PWS team of the Dutch PWS Reference Centre. Cognitive functioning was measured biennially during 8 years of GH treatment by a psychologist experienced in testing children with PWS, in our PWS Reference Centre (Erasmus University Medical Centre/Sophia Children's Hospital, Rotterdam, The Netherlands).

\section{Anthropometry}

Height was measured using a Harpenden stadiometer (Holtain Limited, Crosswell, United Kingdom), weight was measured on a calibrated electronic digital scale (Servo Balance KA-20-150S; Servo Berkel Prior, Katwijk, The Netherlands) and head circumference with a measuring tape. Height, weight, BMI and head circumference were expressed as standard deviation scores (SDS) adjusting for gender and age according to Dutch reference values $(16,17)$ using Growth Analyser 4.0 (available at www. growthanalyser.org). 


\section{Cognition}

Cognitive functioning was assessed by a shortened version of the Wechsler Intelligence Scale for Children (WISC), which is suitable for children between the age of 6 and 16 years (18). The WISC is a well-recognized measure of cognitive ability and demonstrates strong reliability for diagnosis of mild to moderate intellectual disability (19). The subtests Block Design, Vocabulary and Similarities were used. Block Design was used to assess performance IQ, Vocabulary and Similarities were used to assess verbal IQ (20). A shortened version of the WISC was performed because of the limited attention span of children with PWS. Results of the short version of the WISC are correlated with the full-scale IQ test $(21,22,23)$.

All scores were expressed as SDS based on a Dutch population with the same age. Normalized scores ranged from 1 ( -3 SDS) to 19 (+3 SDS), with a mean of 10 (0 SDS). A subtest score between -2 and +2 SDS was considered within the normal range. Calculation of total IQ was performed using the formula: Total IQ $(\mathrm{TIQ})=45.3+2.91 \times$ Vocabulary $\mathrm{SDS}+2.5 \times$ Block Design SDS). This formula has been used in other studies and is based on a Dutch reference population $(24,25)$.

\section{Statistical analysis}

Statistical analyses were performed with the Statistical Package for the Social Sciences 24.0 (SPSS). Baseline data were expressed as median (interquartile range, IQR). Gender and genotypic differences in baseline cognitive functioning and cognitive functioning after 8 years of GH were calculated by Mann-Whitney $U$ tests. Correlations between cognitive functioning and age and head circumference and serum IGF-I levels were calculated by Spearman's correlation analysis.

Changes in cognitive functioning during 8 years were analysed using repeated measurements analysis, with years of GH use ( $0=$ baseline, $24=2$ years of GH treatment, $48=4$ years of GH treatment, $72=6$ years of GH treatment, $96=8$ years of GH treatment) as a categorical independent variable and a compound symmetry covariance matrix. The results of the repeated measurement analyses were expressed as estimated mean (95\% CI). The effects of age, gender, genotype, head circumference and serum IGF-I levels on cognitive functioning during GH treatment were investigated by using these variables as factors (in case of nominal or ordinal variables) or covariates (in case of scale variables) in the model. All subtest scores and TIQ scores were corrected for age, gender and genotype.
Cognitive functioning after 8 years of GH of the children with PWS who started GH during infancy (before the age of 2 years) was compared to the longitudinally studied children with PWS who started GH later in childhood by using unpaired $t$-tests. $P$-values of less than 0.05 were considered statistically significant.

\section{Results}

\section{Baseline clinical characteristics and cognitive functioning}

Forty-three children with PWS (29 girls) who started $\mathrm{GH}$ at a median (IQR) age of 8.1 years $(6.6 ; 11.5)$ were studied during 8 years of continuous GH. Baseline clinical characteristics and cognitive functioning are shown in Table 1. Estimated mean baseline Block Design score was below the lower limit of the reference range of a healthy Dutch population without PWS, while Vocabulary and Similarities subtest scores were within the reference ranges, that is, between -2 and +2 SDS. Estimated mean (95\% CI) baseline TIQ was $66(60 ; 72)$, consistent with a mild mental impairment.

Baseline cognitive functioning was neither different between boys and girls, nor between children with a deletion and a mUPD/imprinting centre defect (ICD)/ translocation $(P>0.12)$. Older age tended to be associated with a lower score on the Similarities subtest $(r=-0.4$, $P=0.05)$. The other subtests and estimated TIQ were not associated with age $(P>0.16)$. Baseline head circumference was positively associated with Block Design SDS $(r=0.41$, $P=0.04)$ and estimated TIQ $(r=0.41, P=0.04)$, but not with Vocabulary and Similarities SDS $(r=0.29, P=0.16$ and $r=0.05, P=0.82$, resp.). Baseline IGF-I tended to be associated with Block Design SDS $(r=0.42, P=0.05)$, but not with the other subtests $(P>0.17)$.

\section{Cognitive functioning during 8 years of GH treatment}

Estimated mean (95\% CI) Block Design SDS changed from $-2.2(-2.6 ;-1.8)$ at start of GH to $-1.8(-2.2 ;-1.4)$ after 8 years of GH $(P=0.18)$ and Similarities SDS from -1.5 $(-2.1 ;-0.9)$ to $-1.3(-1.9 ;-0.7, P=0.66)$. Vocabulary SDS remained similar, being $-1.9(-2.3 ;-1.4)$ at baseline and $-1.9(-2.4 ;-1.5)$ after 8 years $(P=0.85)$. These results demonstrate that visuospatial skills, abstract verbal reasoning and vocabulary skills develop at the same pace as healthy references (Fig. 1). Mean estimated (95\% CI) TIQ changed from $66(60 ; 72)$ to $69(63 ; 75, P=0.57)$. After 8 years of GH, estimated TIQ ranged from 51 to 
Table 1 Clinical characteristics at start of GH treatment in longitudinally studied children with PWS.

\begin{tabular}{l}
\hline Characteristics \\
\hline Number (girls) \\
Genetic subtype, $n$ \\
Deletion \\
mUPD \\
ICD \\
Translocation \\
Unknown \\
Age at start GH treatment (y; median (IQR)) \\
Height (SDS (y); median (IQR)) \\
Weight for height (SDS; median (IQR)) \\
BMI for age (SDS; median (IQR)) \\
Head circumference (SDS; median (IQR)) \\
IGF-I (SDS; median (IQR)) \\
Cognitive functioning \\
(estimated mean (95\% CI)) \\
Block Design (SDS) \\
Vocabulary (SDS) \\
Similarities (SDS) \\
Total IQ
\end{tabular}

\begin{tabular}{c}
\hline Values \\
\hline $43(29)$
\end{tabular}

18

17

4

1

3

$8.1(6.6 ; 11.5)$

$-2.4(-3.5 ;-1.6)$

$1.9(1.2 ; 2.6)$

$1.4(0.7 ; 1.9)$

$-0.6(-0.9 ;-0.2)$

$-1.8(-2.2 ;-1.4)$

$-2.2(-2.6 ;-1.8)$

$-1.9(-2.3 ;-1.4)$

$-1.5(-2.1 ;-0.9)$

$66(60 ; 72)$

$\mathrm{GH}$, growth hormone; ICD, imprinting centre defect; IGF-I, insulin = like growth factor 1; mUPD, maternal uniparental disomy.

94 and $30 \%$ of the children had an estimated TIQ above 70 , the cut-off for mental disability. Overall, cognitive functioning remained similar during 8 years of GH and did not deteriorate.

\section{Influence of clinical and genetic characteristics on cognitive development}

The course of cognitive functioning during 8 years of GH was similar in boys and girls $(P>0.26)$. The course of Block Design SDS during 8 years of GH was significantly different in children with a deletion and children with a $\mathrm{mUPD} / \mathrm{ICD} /$ translocation $(P<0.01)$. In children with a deletion, estimated mean (95\% CI) Block Design SDS changed from $-1.8(-2.3 ;-1.3)$ at baseline to $-1.5(-2.0$; $-1.0)$ after 8 years $(P=0.13)$, while it remained similar in those with a $\mathrm{mUPD} / \mathrm{ICD} /$ translocation, being $-2.4 \mathrm{SDS}$ $(-2.7 ;-2.1)$ at baseline and $-2.5(-2.8 ;-2.2)$ after 8 years $(P=0.76)$. Although Block Design SDS during 8 years of GH did not change significantly in either group, median Block Design SDS after 8 years of GH was significantly higher in the children with a deletion $(P=0.02)$. We found no effect of genotype on the course of the other subtests $(P>0.30)$. Estimated mean $(95 \% \mathrm{CI})$ head circumference SDS increased from $-0.6(-0.9 ;-0.2)$ at baseline to -0.1 $(-0.5 ; 0.2)$ after 8 years $(P<0.01)$ and IGF-I SDS from -1.8 $(-2.2 ;-1.4)$ to $1.7(1.3 ; 2.2, P<0.01)$, but neither the change in head circumference nor the change in IGF-I SDS
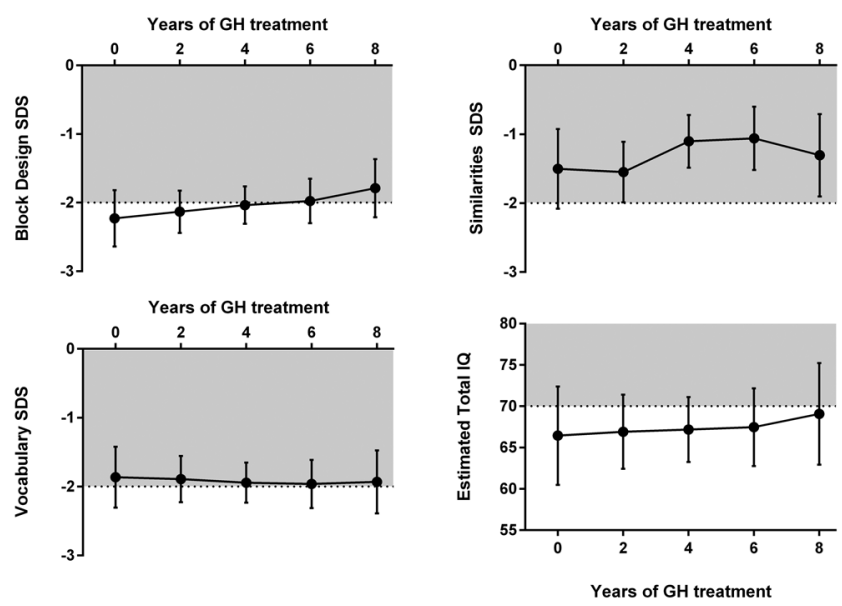

Figure 1

Cognitive functioning during 8 years of $\mathrm{GH}$ in children with PWS tested by WISC. Cognitive functioning during 8 years of GH treatment in children with PWS who started GH later in childhood. The grey rectangles define the normal range of cognitive functioning.

was associated with the change in cognitive functioning during 8 years of GH $(P>0.25)$. A higher IGF-I SDS after 8 years of GH was still associated with a higher Block Design SDS after 8 years of GH $(r=0.43, P=0.04)$. The association between IGF-I and the other subtests after 8 years was not statistically significant $(P>0.14)$.

\section{Effect of age at start of GH on cognitive functioning after 8 years of GH treatment}

To investigate whether starting GH during infancy, that is, before the age of 2 years, would result in better cognitive functioning compared to starting GH later in childhood, we compared WISC results after 8 years of GH of a (separate) group of 22 children from our Dutch PWS cohort who started GH at a median (IQR) age of 1.4 years $(1.0 ; 1.8)$ to those of the 43 longitudinally investigated children who started GH later in childhood (Fig. 2 and Table 2). We could not evaluate the longitudinal effects of 8 years of GH on cognitive functioning in the first group, because WISC is not suitable for children younger than 6 years of age. After 8 years of GH, the 22 children who started GH during infancy were significantly younger and taller than the 43 children who started GH later in childhood $(P<0.02)$. Children who started GH during infancy had a significantly higher Vocabulary SDS $(P<0.01)$ and estimated TIQ score $(P=0.04$; Fig. 2$)$ after 8 years of GH. Scores on the Block Design and Similarities subtests were similar $(P=0.48$ and $P=0.16$, resp.) between the two groups. 


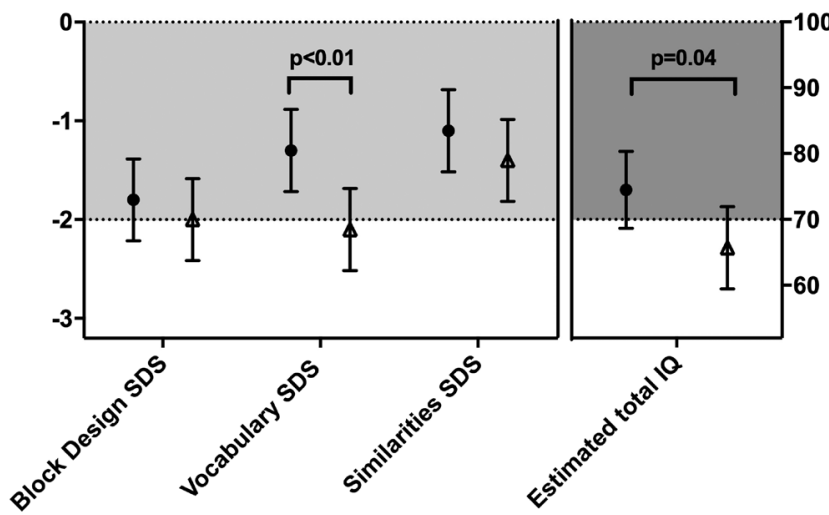

Figure 2

Cognitive functioning after 8 years of GH tested by WISC of the 22 children who started $\mathrm{GH}$ treatment during infancy, before the age of 2 years, compared to 43 longitudinally studied children who started GH later in childhood. Data are presented as mean (S.E.M.). The grey rectangles define the normal range of cognitive functioning, the black dots represent the children who started GH treatment during infancy and the white triangles represent the children who started GH later in childhood.

\section{Discussion}

This is the first long-term study investigating cognitive functioning during 8 years of continuous $\mathrm{GH}$ treatment in children with PWS. Our results demonstrate that the SD scores for visuospatial skills, verbal reasoning skills and vocabulary, as well as estimated TIQ remain similar during 8 years of $\mathrm{GH}$ treatment. This shows that cognitive development in GH-treated children with PWS progresses at the same pace as healthy references and does not deteriorate.

We previously demonstrated in a 2-year randomized controlled trial that cognitive functioning in GH-treated children with PWS developed at the same pace as cognitive functioning in healthy references, while there was a significant deterioration in abstract verbal reasoning and vocabulary in untreated controls (2). In 50 children with PWS who were treated with GH for 4 years, visuospatial skills and abstract verbal reasoning improved significantly (2). In the current study during 8 years of GH treatment, visuospatial skills and abstract verbal reasoning did not improve significantly. Yet, our results are reassuring and they show that GH treatment is able to maintain a similar level of cognitive development compared to healthy references. We should bear in mind that although $\mathrm{GH}$ seems to benefit cognitive functioning, it does so in the context of a developmental disorder with a lower total
Table 2 Clinical characteristics after 8 years of $\mathrm{GH}$. Data are expressed as median (IQR).

\begin{tabular}{|c|c|c|}
\hline Characteristics & $\begin{array}{c}\text { Start of GH } \\
\text { during childhood }\end{array}$ & $\begin{array}{c}\text { Start of GH } \\
\text { during infancy }\end{array}$ \\
\hline Number (girls) & $43(29)$ & $22(9)$ \\
\hline \multicolumn{3}{|l|}{ Genetic subtype } \\
\hline Deletion & 18 & 12 \\
\hline mUPD & 17 & 9 \\
\hline $\mathrm{ICD}$ & 4 & 0 \\
\hline Translocation & 1 & 0 \\
\hline Unknown & 3 & 1 \\
\hline $\begin{array}{l}\text { Age at start GH } \\
\text { treatment (years) }\end{array}$ & $8.1(6.6 ; 11.5)^{1}$ & $1.4(1.0 ; 1.8)$ \\
\hline $\begin{array}{c}\text { Age after } 8 \text { years } \\
\text { of GH (years) }\end{array}$ & $16.0(14.5 ; 19.4)^{1}$ & $9.5(9.0 ; 9.8)$ \\
\hline Height (SDS) & $-0.9(-1.5 ; 0.2)^{1}$ & $0.2(-1.0 ; 1.3)$ \\
\hline Weight for height (SDS) & $1.5(0.3 ; 2.0)$ & $1.3(0.2 ; 1.8)$ \\
\hline Head circumference (SDS) & $0.3(-1.1 ; 1.1)$ & $0.2(0.1 ; 0.5)$ \\
\hline
\end{tabular}

${ }^{1} P<0.02$ compared to 22 children who started GH before 2 years of age. $\mathrm{GH}$, growth hormone; ICD, imprinting centre defect; mUPD, maternal uniparental disomy.

IQ than the general population. Longer duration of $\mathrm{GH}$ treatment, therefore, does not automatically lead to a continuous increase in IQ scores (12).

Because all children with PWS in the Netherlands are treated with GH from a young age, we could not compare cognitive development of GH-treated children with PWS to children with PWS who were not treated with GH. We acknowledge that an RCT would be the first-choice design to investigate the effects of long-term $\mathrm{GH}$ on cognitive functioning in children with PWS, but it would be unethical to withhold GH treatment for 8 years, knowing its positive effects on various outcomes in children with PWS. As our short-term RCT showed a deterioration of cognitive abilities in children with PWS who were not treated with GH during 2 years (2), it is possible that cognitive functioning would have deteriorated if the children in the current study were not treated with $\mathrm{GH}$. The fact that cognitive functioning did not deteriorate and the progress of cognitive development in children with PWS during 8 years of GH treatment was similar to healthy references could therefore be interpreted as a positive result.

How the genetic aberrations underlying PWS lead to cognitive impairment is largely unknown. Studies have shown that it may be related to lower brain volumes and lower cortical complexity, due to alterations in gene networks that are important for early brain development $(26,27)$. Another question that still needs to be elucidated is the mechanism of how GH could affect cognitive functioning. A recent review about the effects of $\mathrm{GH}$ on cognition concluded that $\mathrm{GH}$ might stimulate $\mathrm{GH}$ receptors in brain areas involved in learning and memory, thereby 
improving cognitive functioning (10). It is known that GH receptors are located throughout the brain and that $\mathrm{GH}$ and IGF-I affect the genesis of neurons, thereby stimulating brain growth, development and myelinisation $(8,9)$.

A study in untreated adults with PWS reported a correlation between lower IGF-I levels and poorer intellectual skills (28). Our study found a correlation coefficient of 0.42 between visuospatial skills and serum IGF-I levels $(P=0.05)$. Although there is no unequivocal evidence, there is a possibility that GH treatment affects cognitive functioning by increasing IGF-I levels in patients with PWS. However, more studies need to be performed to confirm this hypothesis. Studies have also shown that GH has direct effects on the CNS that are independent of IGF-I levels (29). Baseline head circumference and visuospatial skills at start of GH were significantly associated. However, the increase in head circumference during GH was not associated with the change in cognitive functioning, demonstrating that, even though head circumference has previously been associated with cognitive functioning, other determinants might be more important for cognitive development and head growth $(25,30,31)$.

After 8 years of GH, children with a deletion had significantly better visuospatial skills compared to those with a mUPD/ICD/translocation. Some studies have also described genotypic differences in performance IQ $(1,2$, $3)$. It is, however, difficult to draw definite conclusions, due to the large variability in cognitive functioning and the small sample-size. After 8 years of GH, estimated TIQ in our study ranged from 51 to 94 and 30\% of children had an estimated IQ above 70 points, the cut-off for mental disability. This broad variety in cognitive functioning has been reported in other studies and underlines the importance of individual assessment of cognitive and social functioning in children with PWS, regardless of the genetic defect. By doing so, we can help identify the difficulties that individual children with PWS face and develop strategies to best deal with these difficulties (27).

Some studies show a correlation between age at start of $\mathrm{GH}$ and the effect of GH on cognitive functioning. The younger the children are at the start of GH treatment, the more they might benefit with regard to psychomotor and cognitive development $(11,12,13)$. GH treatment in children with PWS is started in a critical period of neurodevelopment, which might ameliorate cognitive functioning on the long-term. We found a significantly higher vocabulary and estimated TIQ score after 8 years of GH treatment in the 22 children who started GH treatment during infancy compared to the 43 children who started GH later in childhood. Because WISC is not suitable for children younger than 6 years of age, we could not evaluate cognitive functioning at start of GH treatment and the longitudinal effects of 8 years of $\mathrm{GH}$ in the first group. Nevertheless, our results suggest that an early start of GH during infancy might be beneficial for cognitive development, as reported in previous studies $(11,12,13)$

Combined with an early diagnosis and multidisciplinary support from a very young age, longterm GH treatment during childhood counteracts the clinical course of increasing obesity in PWS and has substantially changed the phenotype of children with PWS. Our study shows that cognitive development during 8 years of GH in children with PWS progresses at the same pace as healthy peers and does not deteriorate. It also shows that vocabulary and estimated TIQ scores after 8 years of GH are higher in children who started GH during infancy - a critical period of neurodevelopment. Further studies are necessary to confirm our findings.

\section{Declaration of interest}

Investigator-initiated study for which $\mathrm{A} \mathrm{H} \mathrm{K}$ received an independent research grant from Pfizer. The other authors have nothing to disclose.

\section{Funding}

This study was an investigator-initiated study investigating the effects of $\mathrm{GH}$ in children with PWS, supported by an independent research grant from Pfizer. Pfizer was neither involved in the conception or design of the study, nor in the collection, analysis or interpretation of data, in writing the manuscript or in the decision to submit the manuscript for publication.

\section{Acknowledgements}

The authors express their gratitude to all the children and parents for their enthusiastic participation in this study. The authors thank M. van Eekelen for all her help and acknowledge E. Snikkers. The authors thank all collaborating pediatric-endocrinologists, pediatricians and other health care providers.

\section{References}

1 Cassidy SB, Schwartz S, Miller JL \& Driscoll DJ. Prader-Willi syndrome. Genetics in Medicine : Official Journal of the American College of Medical Genetics 201214 10-26. (https://doi.org/10.1038/ gim.0b013e31822bead0)

2 Siemensma EP, Tummers-de Lind van Wijngaarden RF, Festen DA, Troeman ZC, van Alfen-van der Velden AA, Otten BJ, Rotteveel J, Odink RJ, Bindels-de Heus GC, van Leeuwen M et al. Beneficial effects of growth hormone treatment on cognition in children with Prader-Willi syndrome: a randomized controlled trial and longitudinal study. Journal of Clinical Endocrinology and Metabolism 201297 2307-2314. (https://doi.org/10.1210/jc.2012-1182)

3 Whittington J, Holland A, Webb T, Butler J, Clarke D \& Boer H. Cognitive abilities and genotype in a population-based sample of people with Prader-Willi syndrome. Journal of Intellectual Disability 
Research 200448 172-187. (https://doi.org/10.1111/j.13652788.2004.00556.x)

4 Bakker NE, Kuppens RJ, Siemensma EP, Tummers-de Lind van Wijngaarden RF, Festen DA, Bindels-de Heus GC, Bocca G, Haring DA, Hoorweg-Nijman JJ, Houdijk EC et al. Eight years of growth hormone treatment in children with Prader-Willi syndrome: maintaining the positive effects. Journal of Clinical Endocrinology and Metabolism 2013 98 4013-4022. (https://doi.org/10.1210/jc.2013-2012)

5 Bakker NE, Kuppens RJ, Siemensma EP, Tummers-de Lind van Wijngaarden RF, Festen DA, Bindels-de Heus GC, Bocca G, Haring DA, Hoorweg-Nijman JJ, Houdijk EC et al. Bone mineral density in children and adolescents with Prader-Willi syndrome: a longitudinal study during puberty and 9 years of growth hormone treatment. Journal of Clinical Endocrinology and Metabolism 2015100 1609-1618. (https://doi.org/10.1210/jc.2014-4347)

6 Festen DA, Wevers M, Lindgren AC, Bohm B, Otten BJ, Wit JM, Duivenvoorden HJ \& Hokken-Koelega AC. Mental and motor development before and during growth hormone treatment in infants and toddlers with Prader-Willi syndrome. Clinical Endocrinology 2008 68 919-925. (https://doi.org/10.1111/j.1365-2265.2007.03126.x)

7 Lo ST, Collin PJ \& Hokken-Koelega AC. Visual-motor integration in children with Prader-Willi syndrome. Journal of Intellectual Disability Research 201559 827-834. (https://doi.org/10.1111/jir.12197)

8 Lai Z, Roos P, Zhai O, Olsson Y, Fholenhag K, Larsson C \& Nyberg F. Age-related reduction of human growth hormone-binding sites in the human brain. Brain Research 1993621 260-266. (https://doi. org/10.1016/0006-8993(93)90114-3)

9 Aberg D. Role of the growth hormone/insulin-like growth factor 1 axis in neurogenesis. Endocrine Development 201017 63-76. (https:// doi.org/10.1159/000262529)

10 Nyberg F \& Hallberg M. Growth hormone and cognitive function. Nature Reviews: Endocrinology 20139 357-365. (https://doi. org/10.1038/nrendo.2013.78)

11 Donze SH, Damen L, Mahabier EF \& Hokken-Koelega ACS. Improved mental and motor development during 3 years of GH treatment in very young children With Prader-Willi syndrome. Journal of Clinical Endocrinology and Metabolism 2018103 3714-3719. (https://doi. org/10.1210/jc.2018-00687)

12 Dykens EM, Roof E \& Hunt-Hawkins H. Cognitive and adaptive advantages of growth hormone treatment in children with PraderWilli syndrome. Journal of Child Psychology and Psychiatry, and Allied Disciplines 201758 64-74. (https://doi.org/10.1111/jcpp.12601)

13 Myers SE, Whitman BY, Carrel AL, Moerchen V, Bekx MT \& Allen DB. Two years of growth hormone therapy in young children with Prader-Willi syndrome: physical and neurodevelopmental benefits. American Journal of Medical Genetics: Part A 2007 143A 443-448. (https://doi.org/10.1002/ajmg.a.31468)

14 Haqq AM, Stadler DD, Jackson RH, Rosenfeld RG, Purnell JQ \& LaFranchi SH. Effects of growth hormone on pulmonary function, sleep quality, behavior, cognition, growth velocity, body composition, and resting energy expenditure in Prader-Willi syndrome. Journal of Clinical Endocrinology and Metabolism $2003 \mathbf{8 8}$ 2206-2212. (https://doi.org/10.1210/jc.2002-021536)

15 Bohm B, Ritzen EM \& Lindgren AC. Growth hormone treatment improves vitality and behavioural issues in children with Prader-Willi syndrome. Acta Paediatrica 2015104 59-67. (https://doi.org/10.1111/ apa.12813)

16 Fredriks AM, van Buuren S, Burgmeijer RJ, Meulmeester JF, Beuker RJ, Brugman E, Roede MJ, Verloove-Vanhorick SP \& Wit JM.
Continuing positive secular growth change in the Netherlands 1955-1997. Pediatric Research 200047 316-323. (https://doi. org/10.1203/00006450-200003000-00006)

17 Fredriks AM, van Buuren S, Wit JM \& Verloove-Vanhorick SP. Body index measurements in 1996-7 compared with 1980. Archives of Disease in Childhood 200082 107-112. (https://doi.org/10.1136/ adc.82.2.107)

18 van Haassen P. Wechsler Intelligence Scale for Children-Revised (Dutch Version), Manual. Lisse, the Netherlands: Swets \& Zeitlinger Publishers BV, 1986.

19 Wechsler D \& WISC IV. Technical and Interpretive Manual. San Antonio, TX, USA: The Psychological Association, 2003.

20 Kaufman A. Essentials of WISC-III and WPPSI-R Assessment. New York: Wiley, 2000

21 Herrera-Graf M, Dipert ZJ \& Hinton RN. Exploring the effective use of the Vocabulary/Block design short form with a special school population. Educational and Psychological Measurement 199656 522-528. (https://doi.org/10.1177/00131644 96056003014)

22 Talkington LW \& Rieker GA. A short form of the WISC for use with the mentally retarded. Psychological Reports 196925 461-462. (https://doi.org/10.2466/pr0.1969.25.2.461)

23 Tsushima WT. Short form of the WPPSI and WPPSI-R. Journal of Clinical Psychology 199450 877-880. (https://doi.org/10.1002/10974679(199411)50:6<877::aid-jclp2270500610>3.0.co;2-r)

24 Hokken-Koelega A, van Pareren Y \& Arends N. Effects of growth hormone treatment on cognitive function and head circumference in children born small for gestational age. Hormone Research 2005 64(Supplement 3) 95-99. (https://doi.org/10.1159/000089324)

25 van Pareren YK, Duivenvoorden HJ, Slijper FS, Koot HM \& HokkenKoelega AC. Intelligence and psychosocial functioning during long-term growth hormone therapy in children born small for gestational age. Journal of Clinical Endocrinology and Metabolism 2004 89 5295-5302. (https://doi.org/10.1210/jc.2003-031187)

26 Lukoshe A, Hokken-Koelega AC, van der Lugt A \& White T. Reduced cortical complexity in children with Prader-Willi syndrome and its association with cognitive impairment and developmental delay. PLoS ONE 20149 e107320. (https://doi.org/10.1371/journal. pone.0107320)

27 Whittington J \& Holland A. Cognition in people with PraderWilli syndrome: insights into genetic influences on cognitive and social development. Neuroscience and Biobehavioral Reviews 201772 153-167. (https://doi.org/10.1016/j.neubiorev.2016.09.013)

28 van Nieuwpoort IC, Deijen JB, Curfs LM \& Drent ML. The relationship between IGF-I concentration, cognitive function and quality of life in adults with Prader-Willi syndrome. Hormones and Behavior 201159 444-450. (https://doi.org/10.1016/j. yhbeh.2011.01.001)

29 Scheepens A, Moderscheim TA \& Gluckman PD. The role of growth hormone in neural development. Hormone Research 2005 64(Supplement 3) 66-72. (https://doi.org/10.1159/000089320)

30 Sammallahti S, Heinonen K, Andersson S, Lahti M, Pirkola S, Lahti J, Pesonen AK, Lano A, Wolke D, Eriksson JG et al. Growth after late-preterm birth and adult cognitive, academic, and mental health outcomes. Pediatric Research 201781 767-774. (https://doi. org/10.1038/pr.2016.276)

31 Hofman MA. Evolution of the human brain: when bigger is better. Frontiers in Neuroanatomy 20148 15. (https://doi.org/10.3389/ fnana.2014.00015)
Received 28 June 2019

Revised version received 13 January 2020

Accepted 20 January 2020 